\title{
Strange freezeout
}

\author{
S. Chatterjee* and R. M. Godbolet \\ Center for High Energy Physics, \\ Indian Institute of Science, \\ Bangalore 560012, India. \\ Sourendu Gupt团 \\ Department of Theoretical Physics, \\ Tata Institute of Fundamental Research, \\ Homi Bhabha Road, Mumbai 400005, India.
}

\begin{abstract}
We argue that known systematics of hadron cross sections may cause different particles to freeze out of the fireball produced in heavy-ion collisions at different times. We find that a simple model with two freezeout points is a better description of data than that with a single freezeout, while still remaining predictive. The resulting fits seem to present constraints on the late stage evolution of the fireball, including the tantalizing possibility that the QCD chiral transition influences the yields at $\sqrt{S}=2700 \mathrm{GeV}$ and the QCD critical point those at $\sqrt{S}=17.3 \mathrm{GeV}$.
\end{abstract}

PACS numbers: 25.75.Dw, 25.75.Nq TIFR/TH/13-15

A fireball is formed in high-energy collision of heavy ions. Within it scattering keeps particles in equilibrium. If the initial collision energy is large enough then this matter could be formed in the plasma phase of the strong interactions [1]. This fireball expands and cools. In the late stages of its evolution, when inelastic scatterings are no longer frequent enough to maintain chemical equilibrium between different hadron species, the system is said to undergo chemical freezeout (CFO) 2]. Ever since the demonstration that data on yields of hadrons for a large range of initial collision energies is explained by a single CFO temperature, $T$, and baryon chemical potential $\mu$, this has been a paradigm for heavy ion collisions 3 [5]. It has been clear that there will be some corrections to this, since the full description of the CFO process must involve kinetic equations, and therefore the densities of hadrons and their reaction cross sections [6]. The expectation was that these corrections are fairly small, and therefore substructure in CFO is a detail. This paradigm is different from decoupling in the early universe, where the hierarchy of interactions gave significantly different $\mathrm{CFO}$ for photons and neutrinos.

However, in a hadronic fireball whose lifetime is short compared to the time-scale of weak interactions, strangeness changing transmutations of baryons occur in interactions with kaons, via intermediate states of excited strange baryons. Furthermore, $\phi$ decays into pairs of kaons $83 \%$ of the time. Although the width of $\phi$ is only $4.3 \mathrm{MeV}$, this is a reflection of reduced phase space volume rather than a reduced interaction strength. This means that the inverse reaction has a low threshold, and, as a result, $\phi$ is in equilibrium with the kaons. However, when kaons decouple, all these hadrons fall out of equilibrium, so providing a natural mechanism for multiple CFO.

Since the pion density is high, and baryon isospin changing reaction thresholds low, isospin transmutations can still proceed [7]. Since baryons of different strangeness each remain connected to pions, they have the same isospin chemical potential even when they are otherwise chemically decoupled. The relative yields of all isodoublets are approximately equal because every sector communicates with a common pion isospin bath. It is impossible to test this simple prediction because the doublets involve uncharged baryons, which are invisible to detectors. Even the comparison of charged members of isotriplets, $\Sigma^{ \pm}$and $\pi^{ \pm}$, is ruled out because $\Sigma^{ \pm}$cannot be reconstructed from its decays since the products include uncharged hadrons.

The paradigmatic case, which we call $1 \mathrm{CFO}$, is merely the simplest of a hierarchy of models because the kaons and pions happen to decouple at the same time. The next simplest possibility, which we call $2 \mathrm{CFO}$, is that all strange particles and $\phi$ decouple together at one time, and all other non-strange particles together at another time. In this paper we examine data on hadron yields 8 26] within the framework of the second model to check whether there is any evidence for a clear separation of pion and kaon CFO. We report that there is indeed widespread evidence for $2 \mathrm{CFO}$ and perhaps some evidence that even more detailed modelling of the chemical kinetics is called for. Multiple freezeouts give us the ability to use hadrons to look back into part of the history of the fireball. We show here that interesting physics can follow from this.

Immediately after CFO, hadrons can be considered to be interacting weakly. Then we can describe this matter as an ideal resonance gas while preserving continuity of thermodynamic variables. We populate this gas with all hadron resonances with masses up to $2 \mathrm{GeV}$ [27], neglect widths and excluded volumes, set the strangeness undersaturation factor $\gamma_{S}$ to unity, but treat the strange and 


\begin{tabular}{|c|c|c|c|c|c|c|c|c|}
\hline $\begin{array}{l}\sqrt{S_{N N}} \\
(\mathrm{GeV})\end{array}$ & Ref & $\begin{array}{c}10^{4} V_{S} \\
\left(\mathrm{MeV}^{-3}\right)\end{array}$ & $\begin{array}{c}10^{4} V_{N S} \\
\left(\mathrm{MeV}^{-3}\right)\end{array}$ & $\begin{array}{c}T_{S} \\
(\mathrm{MeV})\end{array}$ & $\begin{array}{c}T_{N S} \\
(\mathrm{MeV})\end{array}$ & $\begin{array}{c}\mu_{S} \\
(\mathrm{MeV})\end{array}$ & $\begin{array}{c}\mu_{N S} \\
(\mathrm{MeV})\end{array}$ & $\chi^{2} / N_{d f}$ \\
\hline 6.27 & [8-11] & $1.1(0.2)$ & $1.6(0.3)$ & $139(4)$ & $131(4)$ & $435(11)$ & 446 (10) & $1.6 / 4$ \\
\hline 7.62 & [8-11] & $1.2(0.2)$ & $1.4(0.3)$ & $144(3)$ & $139(3)$ & $399(13)$ & 395 (10) & $3.0 / 5$ \\
\hline 7.7 & [15] & $1.0(0.2)$ & $1.5(0.6)$ & $147(3)$ & $138(8)$ & $424(18)$ & $368(28)$ & $8.0 / 4$ \\
\hline 8.76 & [9-12] & $0.8(0.1)$ & $1.3(0.4)$ & $152(3)$ & $145(5)$ & $393(15)$ & $358(18)$ & $4.4 / 5$ \\
\hline 11.5 & [15] & $1.0(0.1)$ & $1.9(0.7)$ & $157(3)$ & $142(7)$ & $310(15)$ & $278(28)$ & $0.8 / 4$ \\
\hline 17.3 & [10-14] & $1.1(0.2)$ & $2.8(0.4)$ & $157(3)$ & $142(3)$ & $214(14)$ & & $15 / 7$ \\
\hline 39. & [15] & $1.0(0.2)$ & $2.4(0.8)$ & $168(4)$ & $148(8)$ & 115 (13) & $98(24)$ & $1.2 / 4$ \\
\hline 62.4 & [16-18] & $1.3(0.3)$ & $2.3(0.7)$ & $169(5)$ & $155(8)$ & $70(20)$ & $65(25)$ & $8.0 / 7$ \\
\hline 130. & [19-22] & $1.6(0.5)$ & $2.5(1.0)$ & $169(6)$ & $157(8)$ & $35(23)$ & $25(20)$ & $4.4 / 5$ \\
\hline 200. & [23-25] & $2.2(0.4)$ & $2.8(0.8)$ & $164(3)$ & $155(6)$ & $31(11)$ & $22(16)$ & $23 / 6$ \\
\hline 2700. & [26] & $4.1(0.6)$ & $8.8(0.8)$ & $162(3)$ & $146(3)$ & $14(12)$ & $-2(7)$ & $4.4 / 6$ \\
\hline
\end{tabular}

TABLE I: The freezeout parameters in 2CFO; the errors indicated are for single parameter variation.
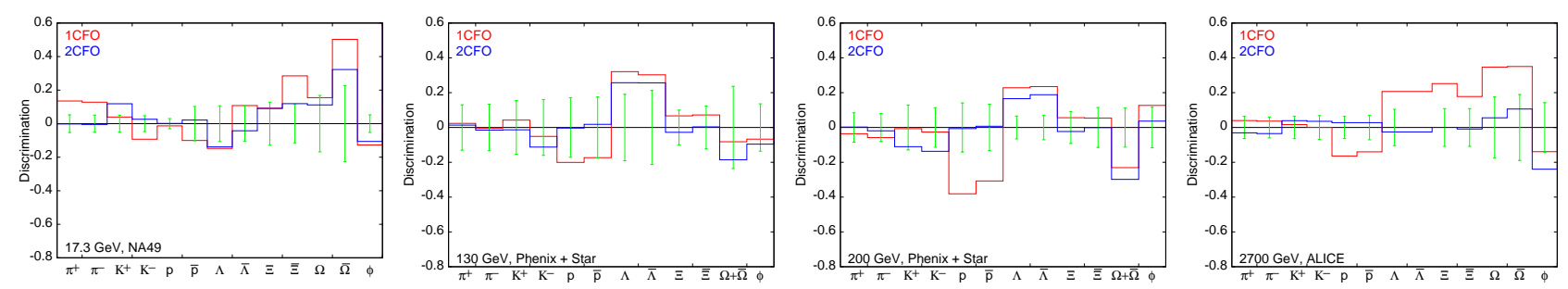

FIG. 1: Discrimination, $\Delta_{h}$, in different models of chemical freezeout, compared to relative error, $R_{h}$.

electric charge fugacities as in [5]. In this model for 1CFO we are able to treat the data on yields at a level of precision similar to that achieved by more detailed models: for example, for the $130 \mathrm{GeV}$ data set from RHIC treated in [28] we find $\chi^{2}=12$, which is close to the quoted value of $\chi^{2}=15$, with similar fitted parameters. On the other hand, even detailed 1CFO models are known to be unable to provide a description of the data at $\sqrt{S}=2700$ $\mathrm{GeV}$ [26]. Consequently, a late stage non-thermal explanation has been put forward [29]. Although this must certainly arise in a full kinetic computation, its strength must be calibrated against the effect of multiple CFOs, since that too must occur.

In $2 \mathrm{CFO}$, we fit yields to the same multi-component hadron resonance gas but with one common temperature, $T_{N S}$, baryon chemical potential, $\mu_{N S}$, and volume, $V_{N S}$, for non-strange hadrons (except $\phi$ ), and a different but common temperature, $T_{S}$, baryon chemical potential, $\mu_{S}$, and volume, $V_{S}$, for strange hadrons and $\phi$. Given a measurement of the yield, $Y_{h}$, of a hadron $h$, with error, $\sigma_{h}$, and a model prediction, $Y_{h}^{m}$, the contribution to $\chi^{2}$ from a specific hadron is

$$
\chi_{h}^{2}=\left[\frac{\Delta_{h}}{R_{h}}\right]^{2},
$$

in terms of the discrimination, $\Delta_{h}=1-Y_{h}^{m} / Y_{h}$, and the relative error, $R_{h}=\sigma_{h} / Y_{h}$. It turns out that in most cases $\chi_{\pi^{ \pm}}^{2}$ and $\chi_{K^{ \pm}}^{2}$ are small. Since this is usually forced by small relative errors in these yields, they give tight constraints on $V_{S}, V_{N S}, T_{S}$ and $T_{N S}$. The baryon yields determine $\mu_{S}$ and $\mu_{N S}$. The best-fit parameters are shown in Table \. In Figure 1 we show $\Delta_{h}$ for the data sets which provide the most stringent tests of the models. It is clear that $2 \mathrm{CFO}$ is a substantially better representation of the data than $1 \mathrm{CFO}$.

It is especially interesting to compare $1 \mathrm{CFO}$ and $2 \mathrm{CFO}$ at LHC in Figure 1. There is clearly a tension between the description of the strange and non-strange baryons in $1 \mathrm{CFO}$, as a result of which the $p / \pi$ ratios seemingly move away from equilibrium values. $2 \mathrm{CFO}$ resolves this tension with separate CFO in the two sectors, as explained earlier. The figure shows that this resolves the problem of some yields seeming to be non-thermal at the LHC. We note that this tension is present, albeit at a statistically milder level, also in the data taken at the other energies. There too the same mechanism improves fits.

There is a smaller remnant problem with $\phi, \Lambda, \bar{\Lambda}$, and $\bar{\Omega}$. Since strangeness changing reactions of baryons have high threshold energies, due to the large masses of particles in the intermediate states, strange baryons could decouple early. A simple 3CFO model of this kind is sufficient to explain the remaining discrepancies. We are aware of the danger that this introduces too many parameters, and therefore over-fits the data. The exercise 


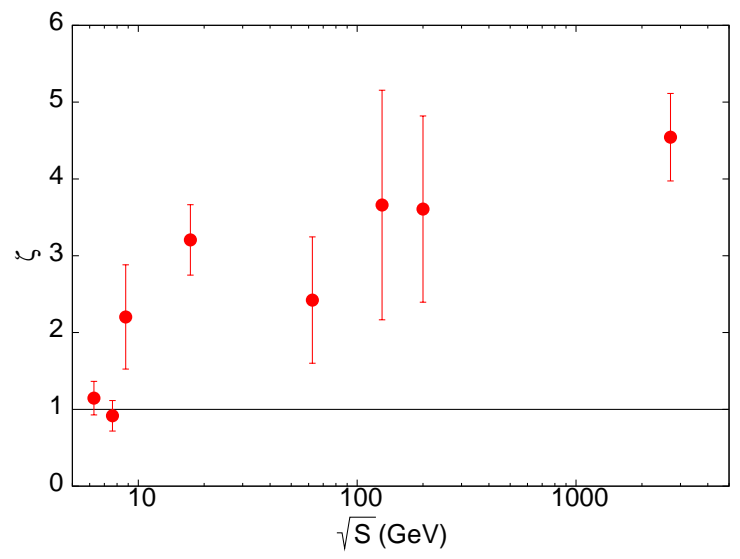

FIG. 2: The evolution of $\zeta=V_{N S} / V_{c}$ with $\sqrt{S}$.

is only meant to illustrate the fact that a detailed kinetic theory computation may improve the description further. A full computation will also include the kinds of mechanisms explored in [29], with the net result that the description will be finer.

We compare $V_{N S}$ with pion correlation volumes, $V_{c}$. Intensity interferometry gives three measures of $V_{c}$, usually denoted by $R_{l}, R_{o}$ and $R_{s}$ [30]. We set $V_{c}=$ $4 \pi R_{l} R_{o} R_{s} / 3$. Although other definitions are possible, the differences are often not statistically significantly. At the two lowest energies the ratio $\zeta=V_{N S} / V_{c}$ is around unity, and rises with $\sqrt{S}$, as shown in Figure 2. The rise can be attributed to the onset of collective expansion. Interestingly, the evidence from $\zeta$ for further enhanced collective flow at the LHC is weak, partly because of the large error bars on $V_{c}$ at RHIC. Any improvement in the experimental errors of the $\sqrt{S}$ dependence of $V_{c}$ would be very useful.

The remaining parameters can be described by the fits

$$
\begin{aligned}
T_{S} & =(150 \pm 6)+\frac{130 \pm 40}{L}-\frac{295 \pm 60}{L^{2}}, \\
\mu_{S} & =-(40 \pm 40)+\frac{85 \pm 300}{L}+\frac{1900 \pm 500}{L^{2}}, \\
T_{N S} & =(142 \pm 7)+\frac{65 \pm 55}{L}-\frac{150 \pm 85}{L^{2}}, \\
\mu_{N S} & =-(85 \pm 20)+\frac{500 \pm 167}{L}+\frac{1000 \pm 250}{L^{2}},
\end{aligned}
$$

where $L=\log \left(\sqrt{S} / M_{p}\right)$ and $M_{p}$ is the proton mass. They are plotted as freezeout curves in Figure 3. At most energies the two CFO surfaces are very close together. However, they move apart at the LHC energy.

It is interesting to speculate on the reason for this. One hypothesis is that this is due to the significantly higher initial energy density, so that an increased pion density delays its CFO. However an argument against this is that the $K / \pi$ ratio remains constant from RHIC to LHC, so the kaon density also rises in proportion, but the kaon

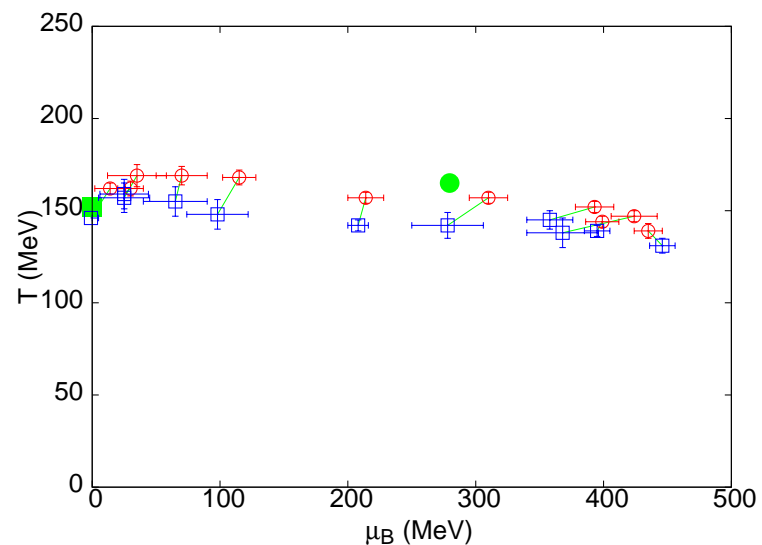

FIG. 3: The freezeout curves for $2 \mathrm{CFO}$ on the phase diagram (circles: strange, squares: non-strange). Points of equal $\sqrt{S}$ are joined by lines. The filled square is the predicted position of the QCD crossover at $\mu=0$ [31] and the filled circle of the QCD critical point 34].

CFO is not delayed proportionately. A qualitatively different explanation relies on the observation that between the two CFOs at very high $\sqrt{S}$, the fireball passes close to the QCD chiral crossover point [31], which is in the critical region of the chiral transition in a theory with $m_{\pi}=0$ [32]. Chiral critical behaviour may then delay pion CFO by lowering the scalar mass. At lower energies, baryon chemical potentials are larger, so the CFOs move away from the chiral critical point. However, data from higher energy runs at the LHC can crucially test this explanation, as the system comes closer to the chiral critical point with increasing $\sqrt{S}$.

Since the two CFOs at the same $\sqrt{S}$ lie on two different points of the same trajectory of fireball evolution, there is more information on late stage evolution than in 1CFO. The $K^{+} / \pi^{+}$ratio peaks at $\sqrt{S}=7.7 \mathrm{GeV}$ [12]. The explanation 33] in $1 \mathrm{CFO}$ is that this occurs at the energy where the fireball crosses from being baryon dominated to meson dominated. This observation continues to hold in $2 \mathrm{CFO}$. However, the new ability to look back into the fireball using different hadrons gives a new piece of information: that the direction of the trajectory changes at this $\sqrt{S}$ (see Figure 31). This divergence of trajectories near the horn would be interesting to understand from hydrodynamics or transport theory.

The opposite seems to happen near the predicted position of the QCD critical point 34]. The comparison of the two trajectories at $\sqrt{S}=11.3$ and $17.3 \mathrm{GeV}$ (see Figure (3) seems to indicate a focusing of trajectories. When trajectories pass near a critical point, hydrodynamic anomalies of this kind are expected [35]. Figure 4 shows that in this energy range there is also some evidence of a broad peak in the ratio $T_{s} / T_{N S}$. A second look at the freezeout curves in Figure 3 indicates that this could be due to a 


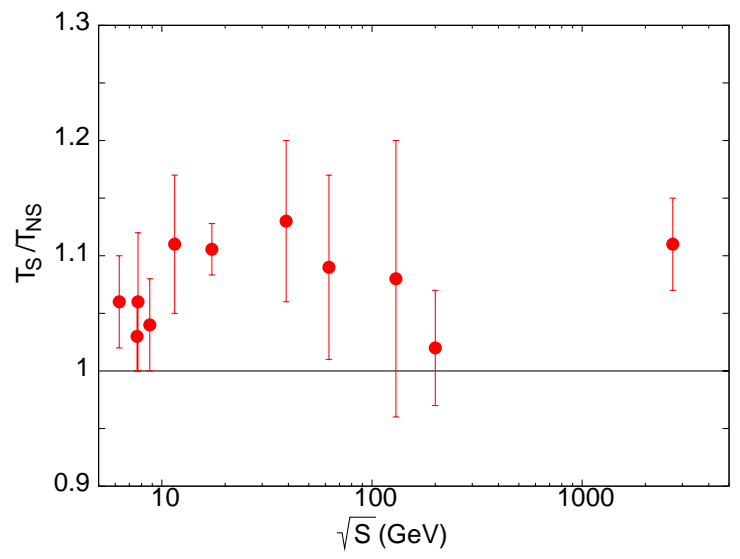

FIG. 4: The ratio $T_{S} / T_{N S}$ as a function of $\sqrt{S}$ possibly has a broad peak in the vicinity of $\sqrt{S}=17.3 \mathrm{GeV}$.

delay in the CFOs. Correspondingly, the fits in eq. (2) indicate that both $T_{S}$ and $T_{N S}$ may reach a minimum in this region. This possibly special behaviour in temperature is not accompanied by any anomaly in $V_{N S}$, as seen from Figure 2 A putative explanation for these observations is a slow expansion in out-of-equilibrium dynamics near the critical point, followed by re-thermalization and delayed freezeout. These mild experimental hints for interesting physics in this region could be critically tested if the beam energy scan at RHIC runs long enough in the region of $\sqrt{S}$ between 19 and $11 \mathrm{GeV}$, and collects enough statistics to study hadron yields more precisely.

In summary, we have shown that there is strong evidence for at least two step CFO of hadrons in the fireball produced by the collision of relativistic heavy-ions, and perhaps even some evidence of further substructure. The separation between CFOs of different hadrons allows us to look back into the fireball with strongly interacting probes during the late stages of its evolution and learn about the physical conditions there. Some of the exciting possibilities which arise are that one may see some signals of the QCD chiral transition at LHC energies, and of the QCD critical point at energies close to that where STAR sees anomalies in the fluctuations of conserved quantities. The LHC is now inadvertently doing a energy scan; a similar very-high statistics scan at the RHIC would be revealing.

We would like to thank Rajeev Bhalerao and Bedanga Mohanty for their comments. RG wishes to thank the Department of Science and Technology, Government of India, for support under grant no. SR/S2/JCB-64/2007. SG would like to thank the Department of Science and Technology, Government of India, for support under grant no. SR/S2/JCB-100/2011.
* Electronic address: sandeep@cts.iisc.ernet,in

† Electronic address: rohini@cts.iisc.ernet.in

‡ Electronic address: sgupta@tifr.res.in

[1] I. Arsene et al., Nucl. Phys. A 757 (2005) 1; B. B. Back et al., Nucl. Phys. A 757 (2005) 28; J. Adams et al., Nucl. Phys. A 757 (2005) 102; K. Adcox et al., Nucl. Phys. A 757 (2005) 184.

[2] U. Heinz, Nucl. Phys. A 685 (2001) 414.

[3] P. Braun-Munzinger, J. Stachel, J. P. Wessels, N. Xu, Phys. Lett. B 365 (1996) 1.

[4] G. D. Yen and M. I. Gorenstein, Phys. Rev. C 59 (1999) 2788.

[5] F. Becattini et al., Phys. Rev. C 64 (2001) 024901.

[6] J. Cleymans, K. Redlich, H. Satz and E. Suhonen, Z. Phys. C 58 (1993) 347; L. P. G. de Assis et al., arXiv:1210.2742

[7] M. Kitazawa and M. Asakawa, Phys. Rev. C 85 (2012) 021901.

[8] C. Alt et al., Phys. Rev. C 77 (2008) 024903.

[9] C. Alt et al., nucl-ex/0512033.

[10] C. Alt et al., Phys. Rev. C 78 (2008) 034918.

[11] C. Alt et al., Phys. Rev. C 78 (2008) 044907.

[12] S. V. Afanasiev et al., Phys. Rev. C 66 (2002) 054902.

[13] C. Alt et al., Phys. Rev. C 73 (2006) 044910.

[14] C. Alt et al., Phys. Rev. Lett. 94 (2005) 192301.

[15] Sabita Das (for the STAR Collaboration) arXiv:1210.6099

[16] B. I. Abelev et al., Phys. Rev. C 79 (2009) 034909.

[17] B. I. Abelev et al., Phys. Rev. C 79 (2009) 064903.

[18] M. M. Aggarwal et al., Phys. Rev. C 83 (2011) 024901.

[19] C. Adler et al., Phys. Rev. C 65 (2002) 041901 (R).

[20] J. Adams et al., Phys. Rev. Lett. 92 (2004) 182301.

[21] K. Adcox et al., Phys. Rev. C 69 (2004) 024904.

[22] K. Adcox et al., Phys. Rev. Lett. 89 (2002) 092302.

[23] J. Adams et al., Phys. Lett. B 612 (2005) 181.

[24] J. Adams et al., Phys. Rev. Lett. 98 (2007) 062301

[25] S. S. Adler et al., Phys. Rev. C 69 (2004) 034909.

[26] Leonardo Milano (for the ALICE Collaboration), arXiv:1302.6624

[27] J. Beringer et al., (Particle Data Group) Phys. Rev. D 86 (2012) 010001.

[28] A. Andronic, P. Braun-Munzinger, J. Stachel, Nucl. Phys. A 772 (2006) 167.

[29] J. Steinheimer, J. Aichelin and M. Bleicher, Phys. Rev. Lett. 110 (2013) 042501; F. Becattini et al., eprint 1212.2431; ibid Phys. Rev. C 85 (2012) 044921.

[30] D. Adamova et al., Nucl. Phys. A 714 (2003) 124; I. G. Bearden et al., Phys. Rev. C 58 (1998) 1656; M. M. Aggarwal et al., Phys. Rev. C 67 (2003) 014906; B. B. Back et al., Phys. Rev. C 73 (2006) 031901; K. Adcox et al., Phys. Rev. Lett. 88 (2002) 192302; C. Adler et al., Phys. Rev. Lett. 87 (2001) 082301; S. S. Adler et al., Phys. Rev. Lett. 93 (2004) 152302; J. Adams et al., Phys. Rev. C 71 (2005) 044906; K. Aamodt et al., Phys. Lett. B 696 (2011) 328.

[31] Y. Aoki et al., J. H. E. P. 0906 (2009) 88.

[32] S. Ejiri et al., Phys. Rev. D 80 (2009) 094505.

[33] J. Cleymans, H. Oeschler, K. Redlich, S. Wheaton, Phys. Lett. B 615 (2005) 50.

[34] R. V. Gavai and S. Gupta, Phys. Rev. D 78 (2008) 114503. 
[35] M. Stephanov, K. Rajagopal and E. Shuryak, Phys. Rev. Lett. 81 (1998) 4816. 